\title{
Time-dependent Hartree-Fock calculations for multinucleon transfer and quasifission processes in the ${ }^{64} \mathrm{Ni}+{ }^{238} \mathrm{U}$ reaction
}

\author{
Kazuyuki Sekizawa ${ }^{1,2, *}$ and Kazuhiro Yabana ${ }^{2,3, \dagger}$ \\ ${ }^{1}$ Faculty of Physics, Warsaw University of Technology, ulica Koszykowa 75, 00-662 Warsaw, Poland \\ ${ }^{2}$ Graduate School of Pure and Applied Sciences, University of Tsukuba, Tsukuba 305-8571, Japan \\ ${ }^{3}$ Center for Computational Sciences, University of Tsukuba, Tsukuba 305-8577, Japan
}

(Dated: May 23, 2016)

\begin{abstract}
Background: Multinucleon transfer (MNT) and quasifission (QF) processes are dominant processes in lowenergy collisions of two heavy nuclei. They are expected to be useful to produce neutron-rich unstable nuclei. Nuclear dynamics leading to these processes depends sensitively on nuclear properties such as deformation and shell structure.
\end{abstract}

Purpose: We elucidate reaction mechanisms of MNT and QF processes involving heavy deformed nuclei, making detailed comparisons between microscopic time-dependent Hartree-Fock (TDHF) calculations and measurements for the ${ }^{64} \mathrm{Ni}+{ }^{238} \mathrm{U}$ reaction.

Methods: Three-dimensional Skyrme-TDHF calculations are performed. Particle-number projection method is used to evaluate MNT cross sections from the TDHF wave function after collision.

Results: Fragment masses, total kinetic energy (TKE), scattering angle, contact time, and MNT cross sections are investigated for the ${ }^{64} \mathrm{Ni}+{ }^{238} \mathrm{U}$ reaction. They show reasonable agreements with measurements. At small impact parameters, collision dynamics depends sensitively on the orientation of deformed ${ }^{238} \mathrm{U}$. In tip (side) collisions, we find a larger (smaller) TKE and a shorter (longer) contact time. In tip collisions, we find a strong influence of quantum shells around ${ }^{208} \mathrm{~Pb}$.

Conclusions: It is confirmed that the TDHF calculations reasonably describe both MNT and QF processes in the ${ }^{64} \mathrm{Ni}+{ }^{238} \mathrm{U}$ reaction. Analyses of this system indicate the significance of the nuclear structure effects such as deformation and quantum shells in nuclear reaction dynamics at low energies.

\section{INTRODUCTION}

It has been known that shell structure and deformation, which are fundamental properties of nuclear structure, play an important role in low-energy heavy ion reactions. For example, the barrier height for nuclear fusion depends on the orientation of colliding nuclei if a deformed nucleus is involved $[1,2]$. In the synthesis of superheavy elements (SHEs), shell effects are crucially important, since they reduce excitation energy of a compound nucleus, and enhancing its survival probability. A remarkable example is the successful synthesis of SHEs by the cold-fusion reactions, where ${ }^{208} \mathrm{~Pb}$ or ${ }^{209} \mathrm{Bi}$ target is utilized [3, 4]. Recently, shell effects on multinucleon transfer (MNT) and quasifission (QF) processes have also been extensively discussed. These reactions are expected to be useful to produce neutron-rich unstable nuclei (see, e.g., Refs. [5-17] and references therein).

The present study aims to elucidate reaction mechanisms of the MNT and QF processes in nuclear reactions involving a heavy deformed nucleus. Specifically, we focus on ${ }^{64} \mathrm{Ni}+{ }^{238} \mathrm{U}$ reaction for which abundant experimental data of both MNT and QF processes are available. Since this system has a large $N / Z$ asymmetry $\left[N / Z=1.29\left({ }^{64} \mathrm{Ni}\right)\right.$ and $\left.1.59\left({ }^{238} \mathrm{U}\right)\right]$, MNT processes toward the charge equilibrium of the total system are expected. A precise measurement of cross sections of the

\footnotetext{
* sekizawa@if.pw.edu.pl

† yabana@nucl.ph.tsukuba.ac.jp
}

MNT processes was carried out by L. Corradi et al. [18]. Moreover, ${ }^{64} \mathrm{Ni}+{ }^{238} \mathrm{U}$ reaction has been expected as a possible candidate for synthesizing a SHE with $Z=120$. To examine this possibility, E.M. Kozulin et al. measured fragment mass and total kinetic energy (TKE) distributions at several incident energies [19]. In Ref. [19], it was shown that mass-symmetric fragments are hardly produced in ${ }^{64} \mathrm{Ni}+{ }^{238} \mathrm{U}$ reaction. This fact indicates a strong suppression of the fusion reaction by QF processes. Massangle and mass-TKE distributions including ${ }^{64} \mathrm{Ni}+{ }^{238} \mathrm{U}$ were reported by J. Tōke et al. [20].

To investigate MNT and QF processes theoretically, various models have been developed. For MNT reactions, semiclassical models called GRAZING [21-23] and Complex WKB [24] have been developed with great successes [25]. The GRAZING has recently been extended to incorporate transfer-induced fission, which is referred to as GRAZING-F [26]. To describe damped collisions, a dynamical model based on Langevin-type equations [9-13, 27-30], the dinuclear system (DNS) model [1417, 31-38], and the improved quantum molecular dynamics model (ImQMD) [39-44] have been extensively developed. Despite numerous successes in describing measurements, they are to some extent empirical, containing adjustable parameters. This fact limits their predictive power. To further extend our understanding of reaction mechanisms and to improve reliability to predict cross sections, we apply the microscopic time-dependent Hartree-Fock (TDHF) theory to MNT and QF processes.

Three-dimensional simulations based on the TDHF theory for low-energy nuclear reactions started around 
70's. They have been successful to describe various phenomena such as fusion reactions and deep inelastic collisions $[45,46]$. Applications of the TDHF theory to $\mathrm{MNT}$ and QF processes are rather new. In Ref. [47], we applied the TDHF theory to investigate MNT processes in ${ }^{40,48} \mathrm{Ca}+{ }^{124} \mathrm{Sn},{ }^{40} \mathrm{Ca}+{ }^{208} \mathrm{~Pb}$, and ${ }^{58} \mathrm{Ni}+{ }^{208} \mathrm{~Pb}$ reactions at energies near the Coulomb barrier, for which precise experimental data are available [48-51]. Applying the particle-number projection (PNP) method [52] to the TDHF wave function after collision, we evaluated transfer probabilities and cross sections for each channel specified by the number of neutrons and protons in the reaction products. From the comparison with measurements, we showed that the TDHF theory reproduces measured cross sections in an accuracy comparable to other existing models. In Ref. [53], we have extended the PNP method to evaluate expectation values of operators. Recently, we applied our method to an asymmetric system, ${ }^{18} \mathrm{O}+{ }^{206} \mathrm{~Pb}$, at energies above the barrier [54].

Recently, QF processes have been investigated by the TDHF theory [55-62]. First, QF dynamics in collisions of two actinide nuclei such as ${ }^{238} \mathrm{U}+{ }^{238} \mathrm{U}$ [55] and ${ }^{232} \mathrm{Th}+{ }^{250} \mathrm{Cf}[56]$ was investigated. In these studies, it has been suggested that QF dynamics depends sensitively on the nuclear orientations, incident energies, and impact parameters. In Ref. [57] in which QF processes of ${ }^{40} \mathrm{Ca}+{ }^{238} \mathrm{U}$ were reported, it has been indicated that shell effects reflecting $Z=82$ and $N=126$ magic numbers have strong influence in tip collisions, while no shell effect is seen in side collisions. In that work, it has also been recognized that contact time is much longer in side collisions than that in tip collisions. The mass-angle distributions (MADs), which are one of the characteristic observables of QF processes, were calculated and compared with experimental data, showing reasonable agreements $[57,59]$.

In this article, we report detailed investigations of MNT and QF processes in ${ }^{64} \mathrm{Ni}+{ }^{238} \mathrm{U}$ reaction performing systematic TDHF calculations. Since the projectile and the target are open shell nuclei, pairing correlations may be important in the collision dynamics. However, we ignore the effect of pairing correlation in this study, since the inclusion of pairing requires much more computational costs which prevent systematic investigations for various initial conditions. Making use of the PNP method, we are able to make detailed comparisons with measurements, including cross sections. In the studies reported so far [55-61], sensitive dependence of QF dynamics on nuclear structure has been suggested. From our detailed analyses of this system, we expect to elucidate clearly those effects of deformation and quantum shells on QF processes.

The article is organized as follows: In Sec. II, we briefly explain the theoretical framework of the TDHF theory and present computational settings. In Sec. III, we present the results of our TDHF calculations. In Sec. III A, we investigate ${ }^{64} \mathrm{Ni}+{ }^{238} \mathrm{U}$ reaction at $E_{\text {c.m. }} \approx$ $307.35 \mathrm{MeV}$. In Sec. III B, incident energy, impact parameter, and orientation dependence of $\mathrm{QF}$ dynamics is investigated. In Sec. IV, we compare the TDHF results with measurements. In Sec. V, summary and conclusion are presented. A part of the results of the present analyses was reported in Ref. [62].

\section{METHOD}

\section{A. TDHF theory}

We briefly explain our theoretical framework. We start with an action,

$$
S=\int_{t_{1}}^{t_{2}}\left[\sum_{i=1}^{A}\left\langle\psi_{i}(t)\left|\mathrm{i} \hbar \partial_{t}\right| \psi_{i}(t)\right\rangle-\mathcal{E}[\rho(t)]\right] d t,
$$

where $\mathcal{E}[\rho(t)]$ denotes an energy density functional $(\mathrm{EDF})$, which is a functional of various densities and is constructed so as to reproduce various properties of finite nuclei and nuclear matter. Applying the stationary condition, $\delta S / \delta \psi_{i}^{*}=0$, we obtain the TDHF equation,

$$
\mathrm{i} \hbar \partial_{t} \psi_{i}(\mathbf{r} \sigma q, t)=\hat{h}[\rho(t)] \psi_{i}(\mathbf{r} \sigma q, t)
$$

where $\mathbf{r}$ and $\sigma$ are spatial and spin coordinates, respectively. $q(=n$ or $p)$ denotes the isospin of $i$ th nucleon. Single-particle wave functions, $\psi_{i}(\mathbf{r} \sigma q, t)$ $(i=1, \cdots, A)$, satisfy the orthonormal relation, $\sum_{\sigma} \int \psi_{i}^{*}(\mathbf{r} \sigma q, t) \psi_{j}(\mathbf{r} \sigma q, t) d \mathbf{r}=\delta_{i j}$. Single-particle Hamiltonian, $\hat{h}[\rho(t)]$, contains a mean-field potential generated by all the nucleons in the system. The many-body wave function is given by a single Slater-determinant composed of the single-particle wave functions,

$$
\Psi\left(\mathbf{r}_{1} \sigma_{1} q_{1}, \cdots, \mathbf{r}_{A} \sigma_{A} q_{A}, t\right)=\frac{1}{\sqrt{A !}} \operatorname{det}\left\{\psi_{i}\left(\mathbf{r}_{j} \sigma_{j} q_{j}, t\right)\right\}
$$

Once the EDF is given, the theory contains no empirical parameters.

In heavy ion reactions, the initial wave function is a Slater determinant composed of single-particle wave functions of projectile and target nuclei in their ground state. They are prepared separately by solving the static Hartree-Fock (HF) equation and are boosted with the relative velocity. The velocity is evaluated assuming the Rutherford trajectory. For a given set of incident energy $E$ and impact parameter $b$, the solution of Eq. (2) is uniquely determined.

We investigate reactions in which binary reaction products are produced. To make comparisons with measurements, we analyze the TDHF wave function at a certain time after collision when the two fragments are sufficiently separated spatially. We calculate such quantities as fragment masses, total kinetic energy loss (TKEL), scattering angle, and MNT cross sections. 


\section{B. Computational settings}

We use our own code of TDHF calculations for heavy ion reactions [62]. In the code, the TDHF equation is solved in real space and real time. Single-particle wave functions are represented on a three-dimensional uniform grid without any symmetry restrictions. The mesh spacing is set to be $0.8 \mathrm{fm}$. We employ the 11-point finitedifference formula for spatial derivatives. The fourthorder Taylor expansion method is utilized for the timeevolution operator with a single predictor-corrector step. The time step is set to be $\Delta t=0.2 \mathrm{fm} / c$. Hockney's method [63] is used to calculate the Coulomb potential in the isolated boundary condition.

A box with $30 \times 30 \times 30$ grid points is used to calculate the ground state of the projectile and target nuclei. A box with $70 \times 70 \times 30$ grid points is used for reaction calculations. We set the incident direction parallel to the $x$-axis and set the impact parameter vector parallel to the $y$-axis. The initial separation distance between centers of the projectile and the target is set to be $24 \mathrm{fm}$ along the incident direction. We stop TDHF calculations when the distance between centers of the reaction products reaches $26 \mathrm{fm}$.

We use Skyrme SLy5 parameter set [64] for the EDF. The ground state of ${ }^{64} \mathrm{Ni}$ has an oblate shape with $\beta \approx 0.12$, while that of ${ }^{238} \mathrm{U}$ has a prolate shape with $\beta \approx 0.27$. We perform TDHF calculations for three initial orientations of ${ }^{238} \mathrm{U}$ : The symmetry axis of ${ }^{238} \mathrm{U}$ is set parallel to the incident direction ( $x$-axis), set parallel to the impact parameter vector ( $y$-axis), and set perpendicular to the reaction plane ( $x y$-plane). We call these three cases as $x-, y$-, and $z$-direction cases, respectively. Since deformation of ${ }^{64} \mathrm{Ni}$ is not very large, we always set the symmetry axis of ${ }^{64} \mathrm{Ni}$ perpendicular to the reaction plane, assuming that the reaction is not affected much by the direction of the deformed ${ }^{64} \mathrm{Ni}$. Figure 1 schematically shows three cases of initial configurations. Since nuclear rotational motion is very slow, we assume that the nuclear orientation at the contact of two nuclei can be well specified by the configurations at the beginning of the TDHF calculations.

\section{TDHF RESULTS}

A. Overview of the reaction at $\mathbf{E}_{\mathrm{c} . \mathrm{m} .} \approx 307.35 \mathrm{MeV}$

In this Subsection, we show results of TDHF calculations for ${ }^{64} \mathrm{Ni}+{ }^{238} \mathrm{U}$ reaction at $E_{\text {c.m. }} \approx 307.35 \mathrm{MeV}$. At around this incident energy, several measurements have been reported [18-20]. Comparisons of the TDHF results with the measurements will be presented in Sec. IV. The calculations are performed for an impact parameter range, $0 \mathrm{fm} \leq b \leq 12 \mathrm{fm}$. We evaluate the frozen HF barrier as described in Ref. [47]. The barrier height is evaluated to be $242.93 \mathrm{MeV}$ for $x$-direction case and 263.97 MeV for $y$-direction case. The incident center-of- (a) $x$-direction

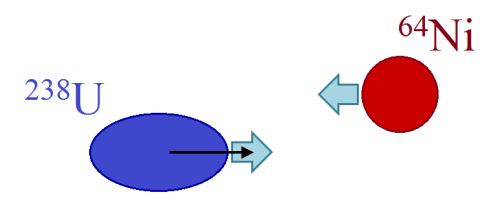

(b) $y$-direction
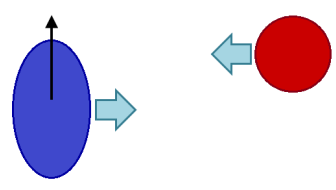

(c) $z$-direction
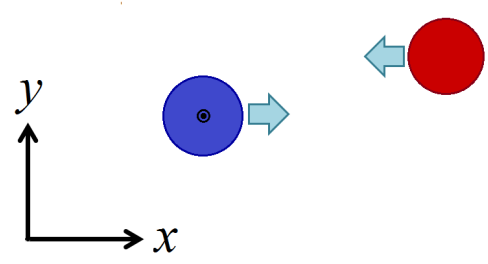

FIG. 1. (Color online) Three initial configurations used for our TDHF calculations of ${ }^{64} \mathrm{Ni}+{ }^{238} \mathrm{U}$ reaction. (a): The symmetry axis of ${ }^{238} \mathrm{U}$ is set parallel to the collision axis ( $x$-axis). (b): The symmetry axis of ${ }^{238} \mathrm{U}$ is set parallel to the impact parameter vector ( $y$-axis). (c): The symmetry axis of ${ }^{238} \mathrm{U}$ is set perpendicular to the reaction plane ( $x y$-plane).

mass energy of $E_{\text {c.m. }} \approx 307.35 \mathrm{MeV}$ corresponds to about $27 \%$ and $16 \%$ above the barrier for the $x$ - and $y$-direction cases, respectively, at $b=0 \mathrm{fm}$. In our TDHF calculations at this incident energy, we always found binary reaction products and no fusion reaction was observed even in head-on collisions.

We will show scattering angle in the center-of-mass frame, $\theta_{\text {c.m. }}$, TKEL, and contact time. The scattering angle and the TKEL are evaluated from the translational motion of reaction products as described in Ref. [47]. The contact time is defined as the duration in which the lowest density between colliding nuclei exceeds a half of the nuclear saturation density, $\rho_{0} / 2=0.08 \mathrm{fm}^{-3}$. The same definition was also used by other authors [57].

In Fig. 2 , we show $\theta_{\text {c.m. }}$. TKEL, and contact time in (a), (b), and (c), respectively, as functions of the impact parameter. Results for $x-, y$-, and $z$-direction cases are shown by red circles, green crosses, and blue open triangles connected with dotted lines, respectively. The same symbols will be used in Figs. 4, 8, 9, and 10. In (a), the scattering angle for the Rutherford trajectory is shown by a dotted curve. In (c), contact time is shown in zeptosecond $\left(1 \mathrm{zs}=10^{-21} \mathrm{sec}\right)$.

We first investigate behavior which does not depend much on the initial orientation of ${ }^{238} \mathrm{U}$. When the impact 


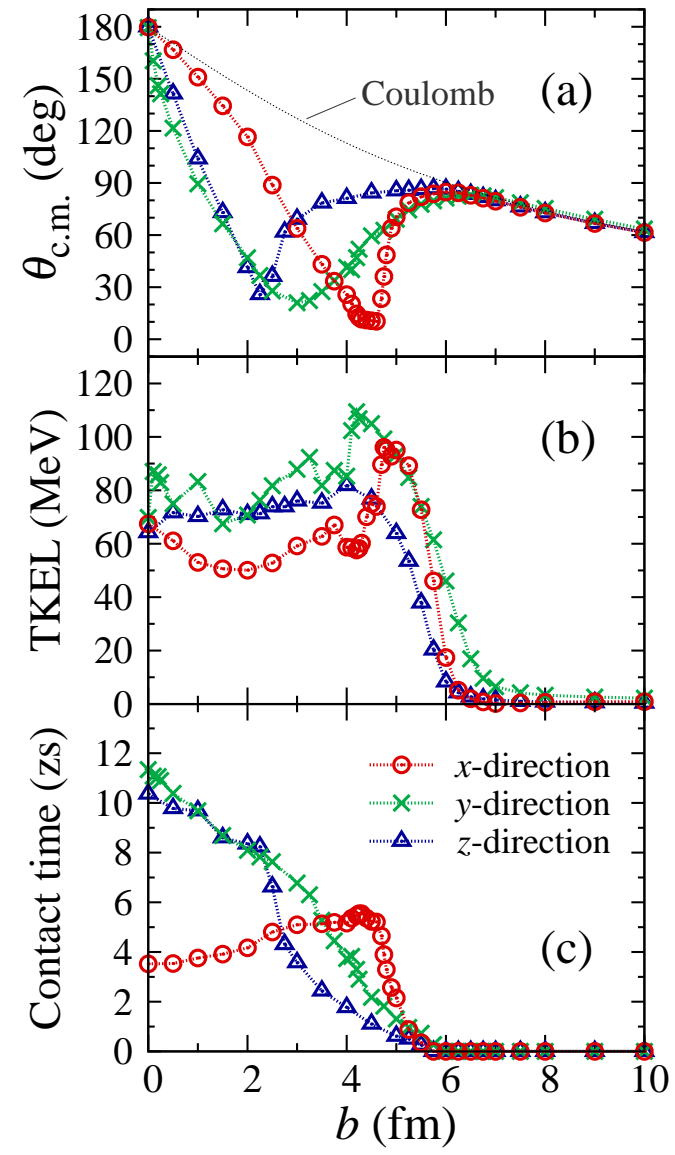

FIG. 2. (Color online) TDHF results for ${ }^{64} \mathrm{Ni}+{ }^{238} \mathrm{U}$ reaction at $E_{\text {c.m. }} \approx 307.35 \mathrm{MeV}$. Scattering angle in the center-of-mass frame, $\theta_{\text {c.m. }}$, total kinetic energy loss (TKEL), and contact time are shown in (a), (b), and (c), respectively, as functions of the impact parameter, $b$. Results for $x$-, $y$-, and $z$-direction cases are shown by red circles, green crosses, and blue open triangles connected with dotted lines, respectively. In (a), the scattering angle for the Rutherford trajectory is shown by a dotted curve.

parameter is sufficiently large $(b \gtrsim 7 \mathrm{fm})$, the reaction is governed by the Coulomb interaction, and the scattering angle coincides with that of the Rutherford trajectory. At this impact parameter region, TKEL is very small and contact time is zero. As the impact parameter decreases $(b \lesssim 7 \mathrm{fm})$, TKEL increases rapidly taking maximum values at $b \approx 4-5 \mathrm{fm}$. Surfaces of two nuclei also start to touch gently, and the nuclear attractive interaction distorts trajectory toward forward angles.

At a small- $b$ region ( $b \lesssim 5 \mathrm{fm}$ ), the contact time becomes substantially long. This indicates a formation of a dinuclear system connected by a thick neck. Because the dinuclear system rotates for a certain period, the scattering angle decreases noticeably as shown in (a). As the impact parameter decreases further, the scattering angle increases monotonically, reaching $180^{\circ}$ (backward scattering) in head-on collisions. In this small- $b$ region, TKEL is roughly constant.

We next look at dependence on the orientation of ${ }^{238} \mathrm{U}$ seen in Fig. 2. As the impact parameter decreases from $b \approx 6 \mathrm{fm}$, we observe a rapid decrease of the scattering angle in (a). In contrast, we observe a rapid increase of the contact time in (c). The decrease (increase) of the scattering angle (contact time) is steepest for the $x$-direction case and becomes moderate as the orientation changes from $x$ - to $y$ - and from $y$ - to $z$-direction. This difference can be understood as follows. In the $x$-direction case, the symmetry axis of ${ }^{238} \mathrm{U}$ is set parallel to the collision axis (Fig. 1 (a)). In this geometry, two nuclei collide substantially at a large impact parameter, $b \approx 5 \mathrm{fm}$, compared to the other cases. In the $z$-direction case, ${ }^{64} \mathrm{Ni}$ always collides with the side of ${ }^{238} \mathrm{U}$ (Fig. 1 (c)). This results in the slowest change of $\theta_{\text {c.m. }}$. and contact time. Results of the $y$-direction case (Fig. 1 (b)) locate between those of the $x$ - and $z$-direction cases.

The contact time shown in (c) has a strong orientation dependence at a small- $b$ region $(b \lesssim 4 \mathrm{fm})$. In the $y$ - and $z$-direction cases, contact time increases monotonically as the impact parameter decreases, reaching 10-11 zs in head-on collisions. On the other hand, in the $x$-direction case, contact time takes almost a constant value (about 4-5 zs), even decreases slightly as the impact parameter decreases. Because of the shorter contact time, the combined dinuclear system does not rotate much. This explains larger scattering angles for the $x$-direction case compared with the other cases at small impact parameters $(b \lesssim 3 \mathrm{fm})$, seen in (a). The observed orientation dependence of the contact time is consistent with the TDHF calculations for ${ }^{40} \mathrm{Ca}+{ }^{238} \mathrm{U}$ reported in [57].

To obtain intuitive understanding of the reaction dynamics, we show in Fig. 3 (a-d) snapshots of the density in the reaction plane for two impact parameters, $5.5 \mathrm{fm}$ and $2 \mathrm{fm}$, and two orientations of ${ }^{238} \mathrm{U}$, the $x$ - and $y$-direction cases. Elapsed time measured from the initial configuration is indicated in zeptosecond. At $b=5.5 \mathrm{fm}$ shown in $(\mathrm{a}, \mathrm{b})$, we find a formation of a thin neck through which a few nucleons are exchanged. The reaction dynamics does not show much difference between the $x$ - and $y$-direction cases at this impact parameter.

Contrarily, we find quite different reaction dynamics at a small- $b$ reaction, $b=2 \mathrm{fm}$, for different orientations of ${ }^{238} \mathrm{U}$. Let us first look at reaction dynamics in the $x$ direction case at $b=2 \mathrm{fm}$ shown in (c). As time evolves, ${ }^{64} \mathrm{Ni}$ collides with ${ }^{238} \mathrm{U}$ at a position close to the tip of the ${ }^{238} \mathrm{U}(t=0.67 \mathrm{zs})$. Then a thick and long neck is developed in the course of the collision, forming an elongated dinuclear system $(t=0.67-2.67 \mathrm{zs})$. After this stage, the neck becomes thinner $(t=3.33-4 \mathrm{zs})$ and eventually ruptures $(t=4.77 \mathrm{zs})$, producing binary reaction products $(t=5.34 \mathrm{zs})$. The produced fragments roughly correspond to ${ }_{40}^{100} \mathrm{Zr}_{60}$ and ${ }_{80}^{202} \mathrm{Hg}_{122}$. We note that we have found very similar shape evolution dynamics to that shown in Fig. 3 (c) in a wide impact parameter range of $b=0-4 \mathrm{fm}$, where the contact time is almost constant as shown in Fig. 2 (c) (see also Supplemental Material [65] for movies of the reactions).

Figure $3(\mathrm{~d})$ shows reaction dynamics in the $y$-direction case at $b=2 \mathrm{fm}$. In this case, ${ }^{64} \mathrm{Ni}$ collides with ${ }^{238} \mathrm{U}$ at a 
(a) $x$-direction, $b=5.5 \mathrm{fm}$

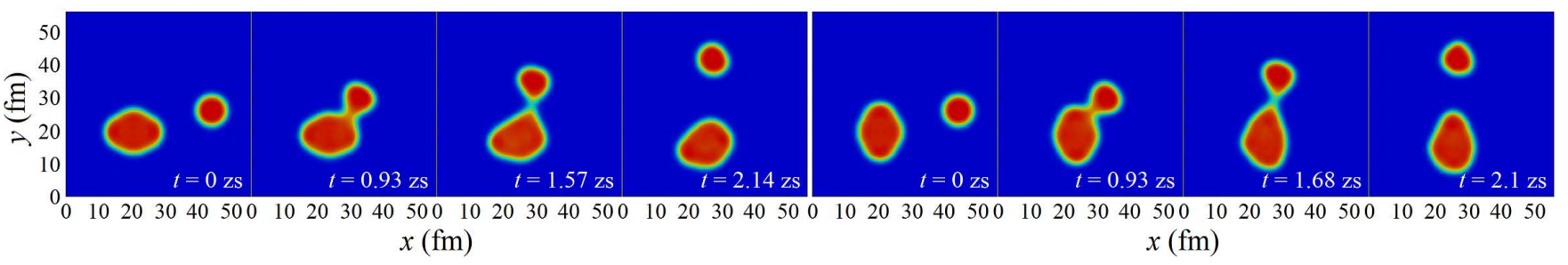

(c) $x$-direction, $b=2 \mathrm{fm}$
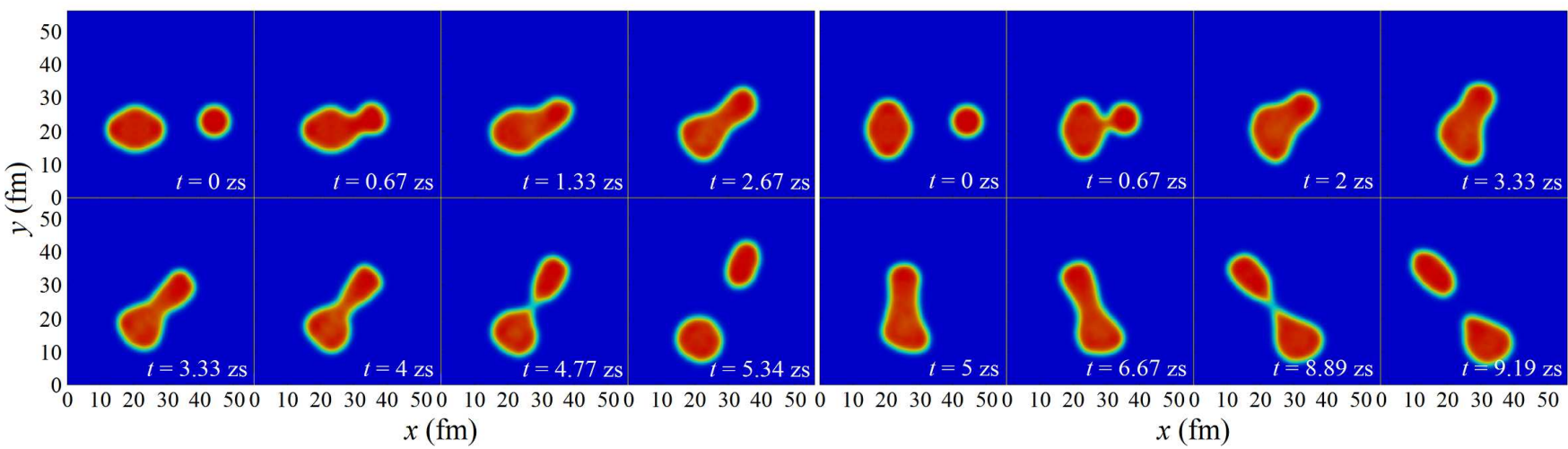

FIG. 3. (Color online) Snapshots of the density in the reaction plane in TDHF calculations for ${ }^{64} \mathrm{Ni}+{ }^{238} \mathrm{U}$ reaction at $E_{\text {c.m. }} \approx$ $307.35 \mathrm{MeV}$. Results for two impact parameters, $b=0.5 \mathrm{fm}$ and $2 \mathrm{fm}$, and two initial orientations of ${ }^{238} \mathrm{U}$, the $x$ - and $y$ direction cases, are shown. Elapsed time measured from the initial configuration is indicated in each panel in zeptosecond $\left(1 \mathrm{zs}=10^{-21} \mathrm{sec}\right)$. See also Supplemental Material [65] for movies of the reactions.

position close to the side of the ${ }^{238} \mathrm{U}(t=0.67 \mathrm{zs})$. After the touch, a somewhat compact composite system with a thick neck structure is formed $(t=2 \mathrm{zs}$ ) (Note that the time of each snapshot is not the same as that shown in (c)). The dinuclear system with a thick neck structure is maintained for a long period and rotates in the reaction plane $(t=2-6.67 \mathrm{zs})$. When the neck ruptures $(t=8.89 \mathrm{zs})$, fragments with more symmetric masses are generated compared with those of the $x$-direction case shown in (c). The produced fragments roughly correspond to ${ }_{47}^{116} \mathrm{Ag}_{69}$ and ${ }_{73}^{185} \mathrm{Ta}_{112}$.

We next investigate average numbers of nucleons in the reaction products as functions of the impact parameter. Figure 4 (a) and (b) show average numbers of neutrons and protons in the lighter fragment, which we denote as $N_{\mathrm{L}}$ and $Z_{\mathrm{L}}$, respectively. Those in the heavier fragment, which we denote as $N_{\mathrm{H}}$ and $Z_{\mathrm{H}}$, are shown in (c) and (d), respectively. $N / Z$ ratios of the lighter and the heavier fragments are also shown in (e). In (e), the fully equilibrated value of the system, 1.52, is indicated by a horizontal dotted line.

When the impact parameter is sufficiently large $(b \gtrsim$ $7 \mathrm{fm}$ ), the average numbers of neutrons and protons coincide with those of the projectile and target nuclei. As the impact parameter decreases $(b \approx 5-6 \mathrm{fm})$, we find that protons are transferred from ${ }^{64} \mathrm{Ni}$ to ${ }^{238} \mathrm{U}$, while neutrons tend to be transferred in the opposite direction. These directions of transfers correspond to those expected form the initial $N / Z$ asymmetry. We show a magnified plot for this impact parameter range as insets in (a-d). The snapshots of the density shown in Fig. 3 (a, b) correspond to reactions in this impact parameter range.

As the impact parameter decreases further, we find a drastic change at around $b \approx 4-5 \mathrm{fm}$. Inside this impact parameter, a mass equilibration process toward the direction increasing the mass symmetry, which we call the mass-drift mode, is observed. In the mass-drift mode, both neutrons and protons are transferred toward the same direction, from the heavier nucleus to the lighter one. While the fragment masses show substantial changes at $b \approx 4-5 \mathrm{fm}$, the $N / Z$ ratios approach monotonically to the fully equilibrated value. From the density profile during the reaction, we find that the shape evolution and the neck rupture are responsible for the mass-drift mode. Once a dinuclear system is formed in the course of collision, the system quickly reaches the charge equilibrium, and the position of the neck rupture determines the amount of transfers of neutrons and protons. In Ref. [47], we reported similar transfer dynamics in lighter systems.

The mass-drift mode observed at $b \lesssim 5$ fm shows noticeable dependence on the initial orientation. In the $z$-direction case (blue open triangles), we find a gradual change of the average number of nucleons. In contrast, in the $x$ - and $y$-direction cases, we observe an abrupt change at $b \approx 4-5 \mathrm{fm}$. In the $x$-direction case (red open circles), the average number of nucleons exhibits a prominent plateau which persists within $0 \mathrm{fm} \leq b \lesssim 4 \mathrm{fm}$. In this impact parameter region, $N_{\mathrm{H}} \approx 120-126$ and 

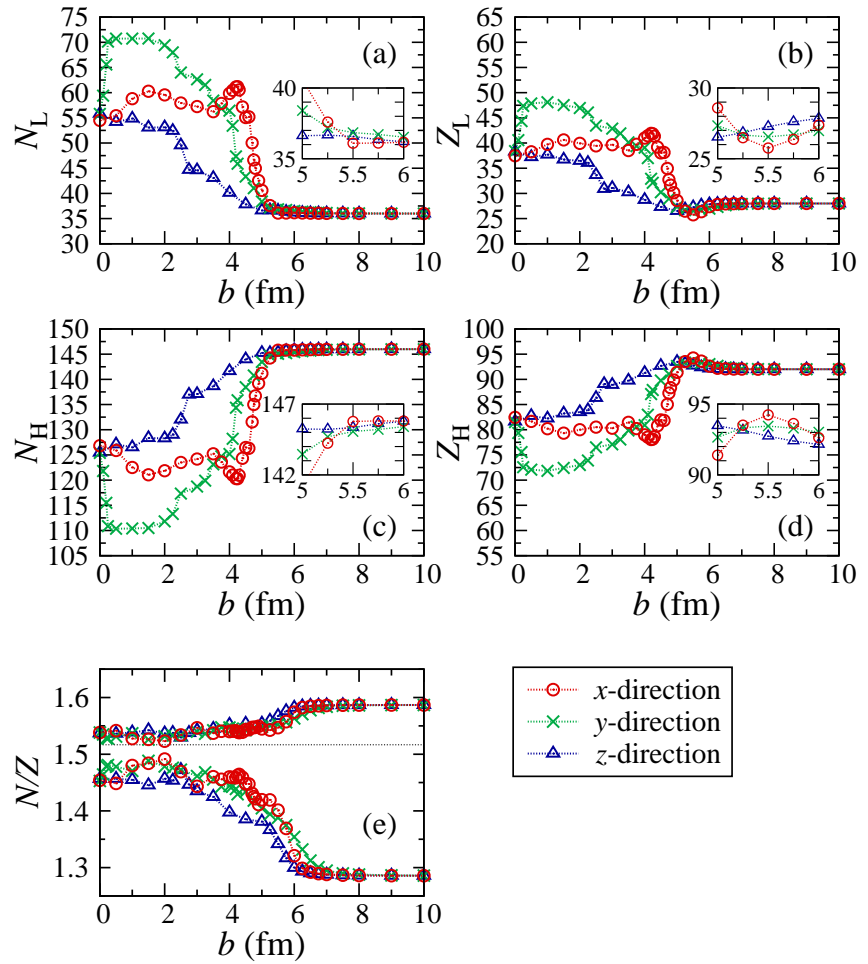

FIG. 4. (Color online) TDHF results for ${ }^{64} \mathrm{Ni}+{ }^{238} \mathrm{U}$ reaction at $E_{\text {c.m. }} \approx 307.35 \mathrm{MeV}$. Average numbers of nucleons in lighter $(\mathrm{a}, \mathrm{b})$ and heavier $(\mathrm{c}, \mathrm{d})$ fragments are shown as functions of the impact parameter, $b$. Left panels $(a, c)$ show those of neutrons, while right panels $(b, d)$ show those of protons. Insets are magnified plots of an impact parameter region, $b=5-6 \mathrm{fm}$. The neutron-to-proton ratios of lighter and heavier fragments are shown in (e). Results for $x-, y$-, and $z$-direction cases are shown by red circles, green crosses, and blue open triangles connected with dotted lines, respectively. In (e), the fully equilibrated value of the system, 1.52, is indicated by a horizontal dotted line.

$Z_{\mathrm{H}} \approx 78-82$ are observed. We consider that the quantum shells of ${ }^{208} \mathrm{~Pb}$ make a significant contribution to this behavior. A similar shell effect of ${ }^{208} \mathrm{~Pb}$ has been reported in the tip collisions of ${ }^{40} \mathrm{Ca}+{ }^{238} \mathrm{U}$ in TDHF calculations [57]. We note that, in our calculations, the lighter partner has $N_{\mathrm{L}} \approx 55-60$ and $Z_{\mathrm{L}} \approx 37-42$. A production of similar fragments has been observed in TDHF calculations for the side collisions of ${ }^{40,48} \mathrm{Ca}+{ }^{238} \mathrm{U}$ at $b=0 \mathrm{fm}$ [58]. A possible influence of stabilization by strongly bound $\mathrm{Zr}$ isotopes with large prolate deformation in this mass region has been advocated [58].

In the $y$-direction case (green crosses), the behavior is quite different. As in the $x$-direction case, we observe an abrupt change of the average number of nucleons at $b \approx 4-5 \mathrm{fm}$. However, the plateau around $N_{\mathrm{H}} \approx 126$ and $Z_{\mathrm{H}} \approx 82$ does not appear. The composite system tends to split into more mass-symmetric fragments. It indicates that the quantum shells of ${ }^{208} \mathrm{~Pb}$ are not significant in this case. A similar interplay between the quantum shells and the nuclear orientation was reported in ${ }^{40} \mathrm{Ca}+{ }^{238} \mathrm{U}$ [57]. In Ref. [57], it was reported that quantum shells do not contribute in the side collisions of ${ }^{40} \mathrm{Ca}+{ }^{238} \mathrm{U}$. Contrarily to it, we find another plateau behavior in the $y$ - and $z$-direction cases. In the $y$-direction case, at a small- $b$ region, $0 \mathrm{fm} \lesssim b \lesssim 2 \mathrm{fm}$, we observe a plateau at around $N_{\mathrm{H}} \approx 110$ and $Z_{\mathrm{H}} \approx 72$ for the heavier fragment and $N_{\mathrm{L}} \approx 70$ and $Z_{\mathrm{L}} \approx 48$ for the lighter fragment. This behavior may be influenced by the shell effect of $Z=50$ in the QF process, although the fragment shows a large deformation as shown in Fig. 3 (d). In the $z$ direction case, a plateau is seen at around $N_{\mathrm{H}} \approx 127$ and $Z_{\mathrm{H}} \approx 83$ for the heavier fragment and $N_{\mathrm{L}} \approx 54$ and $Z_{\mathrm{L}} \approx 37$ for the lighter fragment. This behavior indicates the effect of the quantum shells of ${ }^{208} \mathrm{~Pb}$. We note that influence of quantum shells in QF processes has been routinely observed experimentally [66-72] and discussed theoretically $[10,12,28-30,57,73-76]$.

It is worth emphasizing that, in the $y$-direction case, the average number of nucleons changes dramatically when the impact parameter becomes a tiny but a finite value. For instance, from $b=0$ to $0.25 \mathrm{fm}$, the average number of nucleons changes as large as 25. We consider that the observed behavior is related to the symmetry that appears only at $b=0 \mathrm{fm}$ in which the colliding system has a rotational symmetry around the collision axis. This symmetry disappears once the impact parameter becomes finite.

We note that the behavior at around $b=0 \mathrm{fm}$ is different between $y$ - and $z$-direction cases. To understand the origin of the difference, let us consider shape of the system viewed from a frame rotating with the vector connecting centers of the two colliding nuclei, $\mathbf{R}(t)$, in the adiabatic limit neglecting currents. In the $z$-direction case, the system always persists a reflection symmetry with respect to the plane which contains $\mathbf{R}(t)$ and is perpendicular to the reaction plane. On the other hand, in the $y$-direction case at a nonzero impact parameter, the system does not have the symmetry mentioned above due to the deformed shape of ${ }^{238} \mathrm{U}$. Thus the system may go through more complex shape evolution dynamics. In fact, once the impact parameter becomes nonzero in the $y$ direction case, we find the projectile-like subsystem moving along the elongated direction of the ${ }^{238} \mathrm{U}$-like subsystem forming a very thick neck, which results in the abrupt change of the average number of transferred nucleons (See Supplemental Material [65] for movies of the reactions).

The orientation dependence is also clearly seen in the TKEL at a small- $b$ region $(b \lesssim 4 \mathrm{fm})$ in Fig. 2 (b). In the $y$ - and $z$-direction cases, TKEL takes almost constant values, $\approx 70-80 \mathrm{MeV}$. We observe somewhat larger values of TKEL in the $y$-direction case compared with those of the $z$-direction case. This difference may reflect the reflection symmetry mentioned above which restricts reaction dynamics in the $z$-direction case. In the $x$ direction case, we observe smaller values, $\approx 50-60 \mathrm{MeV}$. In Ref. [77], fission dynamics of ${ }^{258} \mathrm{Fm}$ was investigated by $\mathrm{TDHF}+\mathrm{BCS}$ approach. It was shown that the TKE exhibits clear dependence on the shape of the fissioning nucleus, and that the different shape evolution dynamics 

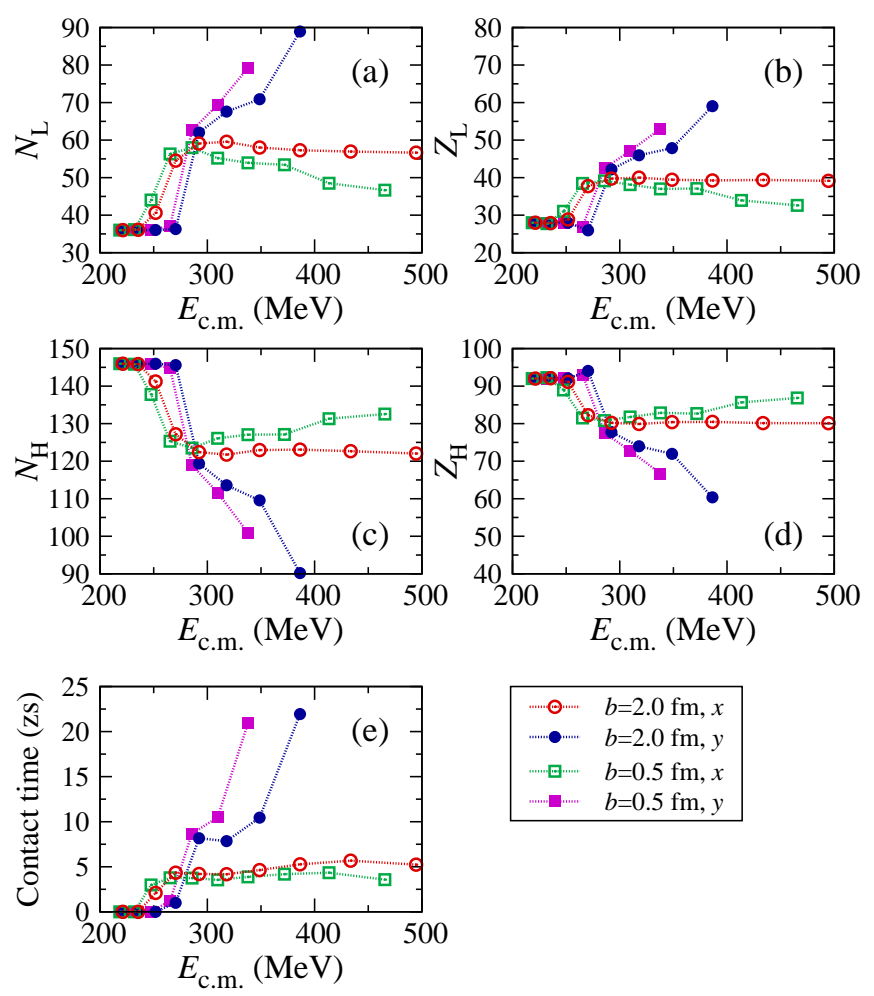

FIG. 5. (Color online) TDHF results for ${ }^{64} \mathrm{Ni}+{ }^{238} \mathrm{U}$ reaction at $b=0.5 \mathrm{fm}$ and $2 \mathrm{fm}$ for $x$ - and $y$-direction cases. Average numbers of nucleons in lighter $(\mathrm{a}, \mathrm{b})$ and heavier $(\mathrm{c}, \mathrm{d})$ fragments are shown as functions of the center-of-mass energy, $E_{\text {c.m. }}$. Left panels $(\mathrm{a}, \mathrm{c})$ show those of neutrons, while right panels $(\mathrm{b}, \mathrm{d})$ show those of protons. In (e), contact time is presented. Results for $b=0.5$ - and 2 -fm cases are shown by squares and circles, and those for $x$ - and $y$-direction cases are shown by open and filled symbols, respectively.

is associated with different valleys in the potential energy surface (PES). Although we have not conducted PES calculations of ${ }_{120}^{302} \mathrm{Ubn}_{182}$ composite system, we expect that there exists a valley in the PES of the composite system associated with the doubly magic ${ }^{208} \mathrm{~Pb}$ and that the valley causes the small TKEL and the short contact time.

We note that an experimentally measured TKE distribution of ${ }^{64} \mathrm{Ni}+{ }^{238} \mathrm{U}$ reaction at a smaller incident energy, $E_{\text {c.m. }} \approx 282.13 \mathrm{MeV}$, was reported [19]. In the measurement, a two-peaked structure of TKE was observed. Although the plot was constructed from selected fragments having $A_{\mathrm{CN}} / 2 \pm 20$, it is expected that different dynamics associated with the large deformation of ${ }^{238} \mathrm{U}$ affects the measured trends.

\section{B. Incident energy dependence}

In this Subsection, we examine incident energy dependence of $\mathrm{QF}$ processes in ${ }^{64} \mathrm{Ni}+{ }^{238} \mathrm{U}$ reaction. We investigate reactions at two impact parameters, $b=0.5 \mathrm{fm}$ and $2 \mathrm{fm}$, for two orientations of ${ }^{238} \mathrm{U}, x$ - and $y$-direction cases.
Figure 5 (a) and (b) show average numbers of neutrons and protons in the lighter fragment, respectively. Those in the heavier fragment are shown in (c) and (d). In (e), contact time is also presented. The horizontal axis is the center-of-mass energy, $E_{\text {c.m. }}$.

First, we consider the $x$-direction case (open symbols). As the center-of-mass energy increases, we find an abrupt change in the fragment masses when the energy exceeds the barrier height, $V_{\mathrm{B}} \approx 242.93 \mathrm{MeV}$. Just above the barrier, the fragment masses are about $N_{\mathrm{L}} \approx 58$ and $Z_{\mathrm{L}} \approx 40$ and $N_{\mathrm{H}} \approx 124$ and $Z_{\mathrm{H}} \approx 80$ for both $b=0.5$ and 2 -fm cases.

For $b=2 \mathrm{fm}$ case (red open circles), the fragment masses are almost independent of the center-of-mass energy for an energy range, $290 \mathrm{MeV} \lesssim E_{\text {c.m. }} \lesssim 500 \mathrm{MeV}$. It indicates a significant influence of the quantum shells of ${ }^{208} \mathrm{~Pb}$, even above barrier energies. On the other hand, for $b=0.5 \mathrm{fm}$ case (green open squares), the amount of transferred nucleons decreases as the center-of-mass energy increases. This behavior implies that the shell effect is weakened as the incident energy increases for $b=0.5 \mathrm{fm}$ case. In the $x$-direction case, an elongated dinuclear system is observed even at energies well above the barrier (See also Supplemental Material [65]). Because of the large elongation, the dinuclear system splits in a relatively short period $(\approx 4-5 \mathrm{zs})$ as seen in Fig. $5(\mathrm{e})$, and no fusion reaction was observed for all incident energies examined here.

Next, we consider the $y$-direction case (filled symbols). For both $b=0.5$ - and 2-fm cases, we observe similar behavior as a function of the center-of-mass energy. As in the $x$-direction case, we find an abrupt change in the fragment masses when the center-of-mass energy exceeds the barrier height, $V_{\mathrm{B}} \approx 263.97 \mathrm{MeV}$. In contrast to the $x$-direction case, the fragment masses continue to change as the center-of-mass energy increases, up to $E_{\text {c.m. }} \approx 338$ (386) $\mathrm{MeV}$ for $b=0.5$ (2) fm. We also find an abrupt change in the contact time in (e). In the $y$-direction case, the composite system shows a compact shape, which becomes a mononuclear shape as the center-of-mass energy increases. The mononuclear system splits into masssymmetric fragments. As a result of the mononuclear system formation, the contact time becomes much longer in the $y$-direction case than that in the $x$-direction case, as shown in (e).

We note that, in the $y$-direction case at higher centerof-mass energies, $E_{\text {c.m. }} \gtrsim 338$ (386) $\mathrm{MeV}$ for $b=0.5$ (2) fm, a capture process takes place, forming a superheavy composite system with $Z=120$. We continued time-evolution calculations up to $40 \mathrm{zs}$ (60,000 time steps). Similar criteria for fusion were also used by other authors $[57,58]$. In this period, the composite system exhibits a compact mononuclear shape (See also Supplemental Material [65]). In Ref. [19], measured fragment mass distributions in ${ }^{64} \mathrm{Ni}+{ }^{238} \mathrm{U}$ reaction were reported at several incident energies. They showed that masssymmetric fragments are hardly produced in the reaction. Our results are consistent with the experimental observation, since the highest incident energy of the experiment 


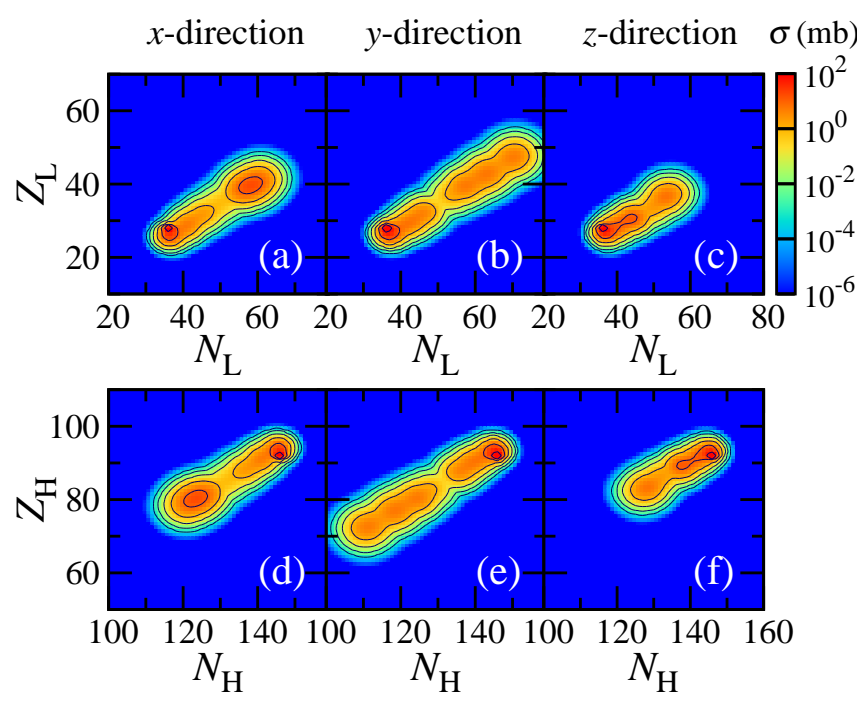

FIG. 6. (Color online) Primary production cross sections, $\sigma(N, Z)$, for ${ }^{64} \mathrm{Ni}+{ }^{238} \mathrm{U}$ reaction at $E_{\text {c.m. }} \approx 307.35 \mathrm{MeV}$ in TDHF calculation. Upper panels (a-c) show cross sections for lighter fragments, while lower panels (d-f) show those for heavier fragments. Contributions from $x$-, $y$-, and $z$-direction cases are shown in left, middle, and right panels, respectively. The contour lines correspond to $\sigma=100,10,1,0.1$, and $0.01 \mathrm{mb}$.

was $E_{\text {c.m. }} \approx 301.05 \mathrm{MeV}$, and is much smaller than the present threshold energy for fusion in our TDHF calculations. Our results indicate that more mass-symmetric fragments will be produced after forming a mononuclear system at higher incident energies, although it should accompany substantial excitation energy. We note that recent experimental data $[78,79]$ show that the superheavy element with $Z=120$ could be formed by ${ }^{64} \mathrm{Ni}+{ }^{238} \mathrm{U}$ reaction at $E_{\text {c.m. }} \approx 332.88 \mathrm{MeV}$, which lived longer than $10^{-18} \mathrm{~s}$.

\section{COMPARISON WITH MEASUREMENTS}

\section{A. Production cross sections}

To compare with measured cross sections of MNT processes, we employ the PNP method $[47,52,53]$. We use the PNP operator,

$$
\hat{P}_{n}^{(q)}=\frac{1}{2 \pi} \int_{0}^{2 \pi} e^{\mathrm{i}\left(n-\hat{N}_{V}^{(q)}\right) \theta} d \theta,
$$

where $\hat{N}_{V}^{(q)}$ is the number operator in a volume $V$. The probability that $n$ nucleons are included in $V$ is given by

$$
P_{n}^{(q)}=\frac{1}{2 \pi} \int_{0}^{2 \pi} e^{\mathrm{i} n \theta} \operatorname{det} \mathcal{B}^{(q)}(\theta) d \theta
$$

where

$\mathcal{B}_{i j}^{(q)}(\theta)=\sum_{\sigma} \int \psi_{i}^{*}(\mathbf{r} \sigma q) \psi_{j}(\mathbf{r} \sigma q)\left(\Theta_{\bar{V}}(\mathbf{r})+e^{-\mathrm{i} \theta} \Theta_{V}(\mathbf{r})\right) d \mathbf{r}$.
$\Theta_{V(\bar{V})}(\mathbf{r})$ denotes a space division function which is equal to 1 inside $V(\bar{V})$ and 0 elsewhere. $\bar{V}$ is the complement of $V$. In practice, the integral in Eq. (5) is evaluated using the trapezoidal rule discretizing the interval into 300 equal grids. The production cross section for a reaction product composed of $N$ neutrons and $Z$ protons is given by

$$
\sigma(N, Z)=2 \pi \int_{0}^{\infty} b P_{N, Z}(b) d b
$$

where $P_{N, Z}$ takes a product from, $P_{N}^{(n)} P_{Z}^{(p)}$, in the TDHF theory.

In Fig. 6, we show production cross sections, $\sigma(N, Z)$, for ${ }^{64} \mathrm{Ni}+{ }^{238} \mathrm{U}$ reaction at $E_{\text {c.m. }} \approx 307.35 \mathrm{MeV}$. Upper panels (a-c) show cross sections for lighter fragments, while lower panels (d-f) show those for heavier fragments. We show cross sections for $x$-, $y$-, and $z$-direction cases in left, middle, and right panels, respectively. To compare with measurements, we should take a proper average over the orientations of ${ }^{238} \mathrm{U}$. We did not do it, since it requires too much computational costs.

From the figure, we find that the cross sections extend widely in the $N-Z$ plane. There is a peak of $\sigma(N, Z)$ at around $\left(N_{\mathrm{L}}, Z_{\mathrm{L}}\right)=(36,28)$ in (a-c) for lighter fragments and $\left(N_{\mathrm{H}}, Z_{\mathrm{H}}\right)=(146,92)$ in (d-f) for heavier fragments. They are contributed from a large- $b$ region, $b \gtrsim 5 \mathrm{fm}$. We also find a peak in $\sigma(N, Z)$ located inside a region of $N_{\mathrm{L}}>$ $50, Z_{\mathrm{L}}>30$ in (a-c) and $N_{\mathrm{H}}<130, Z_{\mathrm{H}}<90$ in (d-f). They are produced by the $\mathrm{QF}$ processes accompanying a large mass-drift toward the mass symmetry, which take place in a small- $b$ region, $b \lesssim 4 \mathrm{fm}$. The appearance of separated peaks in the $N-Z$ plane is caused by the abrupt change of the reaction mechanism from quasielastic and MNT to $\mathrm{QF}$ at $b \approx 4-5 \mathrm{fm}$. The peak positions are consistent with the observation in Fig. 4.

In Ref. [18], experimentally measured transfer cross sections for ${ }^{64} \mathrm{Ni}+{ }^{238} \mathrm{U}$ reaction at $E_{\text {c.m. }} \approx 307.35 \mathrm{MeV}$ were reported. In Fig. 7, we show a comparison of transfer cross sections between our TDHF results and the measurements as a function of the mass number of lighter fragments. Each panel shows cross sections for different proton-transfer channel. The number of transferred protons is indicated by $( \pm x \mathrm{p} ; \mathrm{X})$, where $\mathrm{X}$ stands for the corresponding element. The plus sign is for transfer processes from ${ }^{238} \mathrm{U}$ to ${ }^{64} \mathrm{Ni}$ (pickup), while the minus sign is for the opposite direction (stripping). Measured cross sections are shown by red filled circles. TDHF results in $x$-, $y$-, and $z$-direction cases are shown by red solid, green dashed, and blue dotted histograms, respectively. Cross sections calculated by the GRAZING code [23] using standard input parameters ${ }^{1}$ are also shown by filled areas.

1 Input parameters that we used for the GRAZING calculation: For low-lying excitations: $E_{2}=1.35(0.04) \mathrm{MeV}, B\left(E_{2}\right)=0.076$ $(12.09) e^{2} \mathrm{~b}^{2}, E_{3}=3.56(0.73) \mathrm{MeV}, B\left(E_{3}\right)=0.022(0.58) e^{2} \mathrm{~b}^{2}$; for giant resonances: $E_{2}=57$ (94) $A^{-1 / 3} \mathrm{MeV}$, strength $=0.8$ 


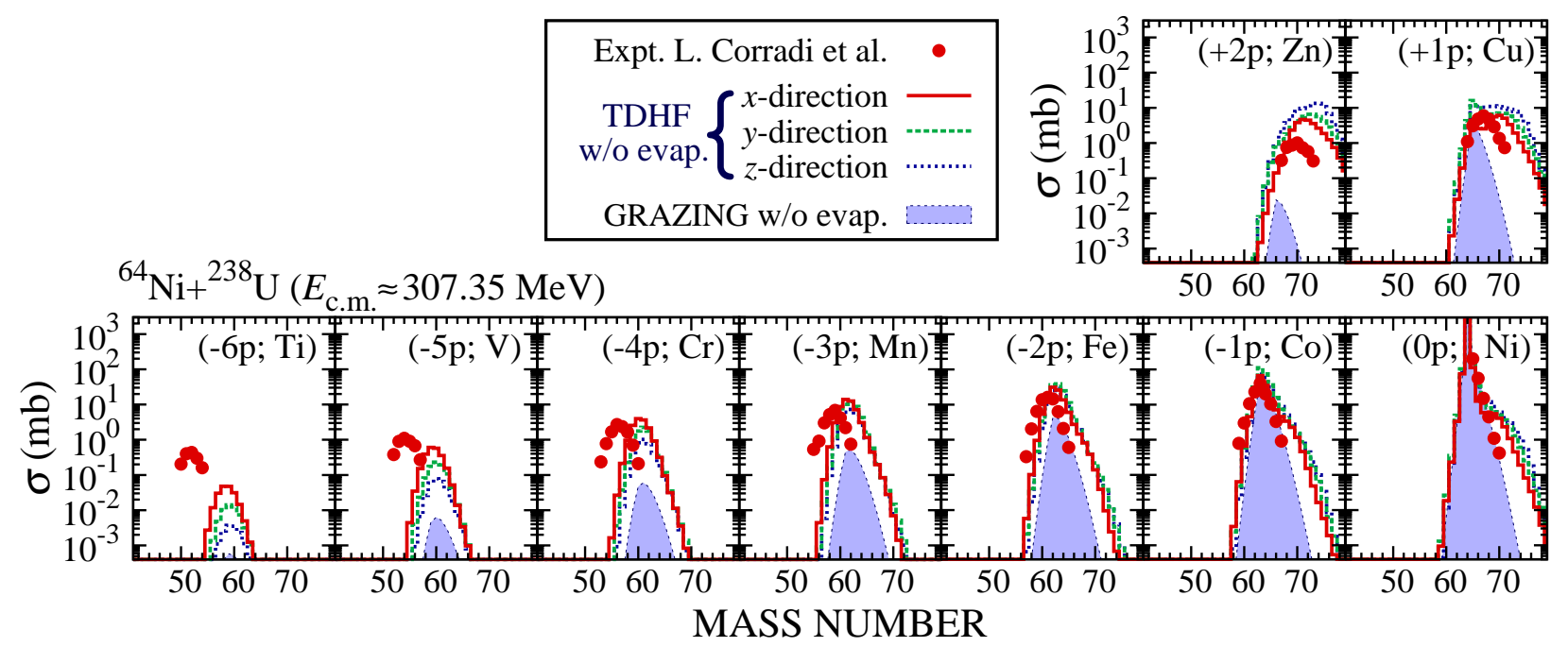

FIG. 7. (Color online) Transfer cross sections for ${ }^{64} \mathrm{Ni}+{ }^{238} \mathrm{U}$ reaction at $E_{\mathrm{c} . \mathrm{m} .} \approx 307.35 \mathrm{MeV}$. Each panel shows cross sections for different proton-transfer channel indicated by $( \pm x \mathrm{p} ; \mathrm{X})$, where $\mathrm{X}$ stands for the corresponding element. The horizontal axis is the mass number of lighter fragments. Experimental data [18] are shown by red filled circles. TDHF results in $x$-, $y$-, and $z$-direction cases are represented by red solid, green dashed, and blue dotted histograms, respectively. We also show cross sections calculated by the GRAZING code [23] with standard parameter sets ${ }^{1}$.

We note that experimental data are suffered by effects of particle evaporation from excited reaction products, whereas the TDHF results correspond to primary cross sections just after the reseparation. In addition, the measurement was performed for an angular range of $50^{\circ} \leq \theta_{\text {lab }} \leq 105^{\circ}$ to cover the main transfer channels in grazing reactions, whereas the TDHF results are obtained by Eq. (7) without filtering by the scattering angle, as in Fig. 6.

From the figure, we find that the TDHF results reasonably reproduce measured cross sections for $(0 p),( \pm 1 p)$, and $(-2 \mathrm{p})$ channels. As the number of removed protons increases $((-x \mathrm{p})$ with $x \geq 3)$, the peak position of experimental cross sections shifts toward less mass (neutron) numbers, which is not reproduced by the TDHF results. The disagreement may partly be originated from the evaporation effect. We note that, although the peak position is different, the height of the peaks of the cross sections for proton-stripping channels is in good agreement with the experimental data, up to $(-4 \mathrm{p})$ channels.

Similar disagreement was observed in lighter systems $[47,54,80,81]$. In Refs. [54, 80, 81], we investigated particle evaporation effects on MNT cross sections using a statistical model. Although the inclusion of the evaporation effect improved the agreement between TDHF results and measurements, there remain disagreements for channels involving a number of transferred nucleons far from the average values in the TDHF calculation. This

(0.4) $\%$ of sum rule, width $=2.5(6)$; for single-particle states: $\delta^{\nu}=8, \delta^{\pi}=8$, level density $=2.455(2.053) \mathrm{MeV}^{-1}$ (neutron), 10.527 (8.298) $\mathrm{MeV}^{-1}$ (proton); these values are for projectile (target). failure indicates a necessity of descriptions beyond the TDHF theory. Beyond mean-field theories such as the method of Balian and Vénéroni $[82,83]$ and the stochastic mean-field approach [84-90] have recently been developed, which are expected to remove the discrepancies.

In Fig. 7, we find that the cross sections depend rather weakly on the initial orientation of ${ }^{238} \mathrm{U}$. Difference is substantial only for $(-5 \mathrm{p}),(-6 \mathrm{p})$, and $(+2 \mathrm{p})$ channels. Differences in these channels are associated with the different trends of nucleon transfer. The proton-stripping processes are originated from an impact parameter region, $b \approx 5-6 \mathrm{fm}$, as shown in Fig. 4. From the insets shown in Fig. 4 (b, d), we find that proton-stripping processes are favored in the $x$-direction case. This trend results in the difference in $(-5 \mathrm{p})$ and $(-6 \mathrm{p})$ channels. In Fig. 4, a gradual change of the average number of nucleons was observed in the $z$-direction case. This change brings a large contribution to $(+1 \mathrm{p})$ and $(+2 \mathrm{p})$ channels from a wide impact parameter range.

From a comparison between cross sections by TDHF and those by GRAZING, we find that the TDHF results show a better overall agreement with experimental data. It is remarkable that the TDHF calculation provides substantial cross sections for the proton-pickup channels. The GRAZING calculation underestimates cross sections for those channels, especially for $(+2 p)$ channel, for which the TDHF calculation overestimates. In Ref. [18], it was mentioned that lighter fragments with proton numbers up to $Z \approx 40$ were observed experimentally, especially at forward angles, although quantitative cross sections were not shown. The TDHF calculation provides substantial cross sections for lighter fragments with $Z \approx 40$ (cf. Fig. $6(\mathrm{a}-\mathrm{c})$ ) as a result of the QF processes at a small- $b$ region, $b \lesssim 4 \mathrm{fm}$. 


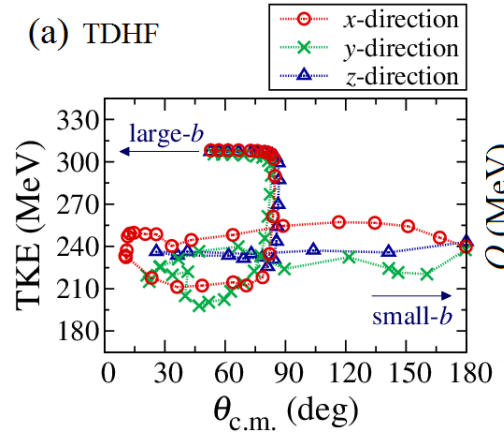

(b) Expt. L. Corradi et al.

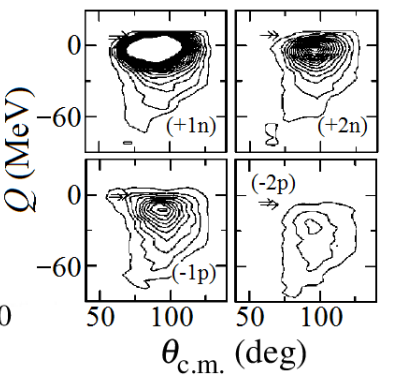

FIG. 8. (Color online) Wilczyński plots. (a): TDHF results for ${ }^{64} \mathrm{Ni}+{ }^{238} \mathrm{U}$ reaction at $E_{\text {c.m. }} \approx 307.35 \mathrm{MeV}$. Results for $x$-, $y$-, and $z$-direction cases are shown by red circles, green crosses, and blue open triangles connected with dotted lines, respectively. (b): Experimental data of Wilczyński plots for main transfer channels, $(+1 \mathrm{n}),(+2 \mathrm{n}),(-1 \mathrm{p})$, and $(-2 \mathrm{p})$, in ${ }^{64} \mathrm{Ni}+{ }^{238} \mathrm{U}$ reaction at $E_{\text {c.m. }} \approx 307.35 \mathrm{MeV}$. The figures shown in (b) are taken from Ref. [18].

\section{B. Wilczyński plot}

Combining $\theta_{\text {c.m. }}$ in Fig. 2 (a) and TKEL in Fig. 2 (b), we obtain the Wilczyński plot which is shown in Fig. 8 (a). In Ref. [18], experimental data of Wilczyński plots for various transfer channels were reported for grazing reaction of ${ }^{64} \mathrm{Ni}+{ }^{238} \mathrm{U}$ at $E_{\text {c.m. }} \approx 307.35 \mathrm{MeV}$. In (b), we show the experimental data for main transfer channels, $(+1 \mathrm{n}),(+2 \mathrm{n}),(-1 \mathrm{p})$, and $(-2 \mathrm{p})$.

The experimental data show a peak at around $\theta_{\text {c.m. }} \approx$ $90^{\circ}$, which shifts toward $Q \approx-60 \mathrm{MeV}$ (lower TKE), as the number of transferred protons increases. Our results agree with the observed trend. At the scattering angle of $\theta_{\text {c.m. }} \approx 80^{\circ}-85^{\circ}(b \approx 5-6 \mathrm{fm})$, our TDHF calculation describes proton-stripping processes, as shown in Sec. III A. In this regime, two nuclei touched gently at the distance of closest approach, forming a subtle neck which persists only for a short period. Due to the formation of the subtle neck, nucleons are exchanged between the projectile and target nuclei and the TKE decreases rapidly, while the scattering angle is kept almost constant, $\theta_{\text {c.m. }} \approx 80^{\circ}-85^{\circ}$. In the experimental data shown in (b), we find a tail of the yields which extends toward forward angles up to $\theta_{\text {c.m. }} \approx 75^{\circ}$, as the energy loss increases up to $Q \approx-75 \mathrm{MeV}$. In the TDHF results shown in (a), a similar trend is observed at $\theta_{\text {c.m. }} \approx 75^{\circ}$.

\section{Mass-angle distribution}

QF processes are known to show a characteristic correlation between fragment masses and scattering angle, and thus, MADs in QF processes have been measured extensively [20, 57, 59, 91-93]. The MAD for ${ }^{238} \mathrm{U}+{ }^{64} \mathrm{Ni}$ reaction at $E_{\text {c.m. }} \approx 302.62 \mathrm{MeV}$ was reported by J. Tōke et al. [20]. We compare the TDHF results with the experimental data. Similar comparisons of MADs be-
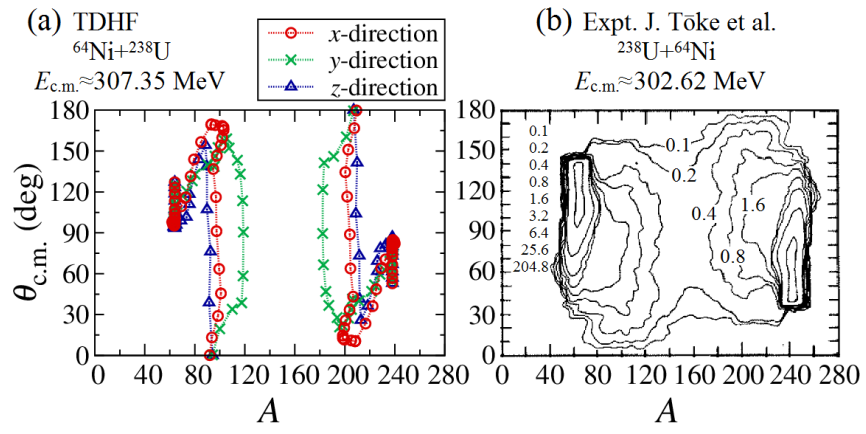

FIG. 9. (Color online) Mass-angle distribution (MAD) plots. (a): TDHF results for ${ }^{64} \mathrm{Ni}+{ }^{238} \mathrm{U}$ reaction at $E_{\text {c.m. }} \approx$ 307.35 MeV. Results for $x$-, $y$-, and $z$-direction cases are shown by red circles, green crosses, and blue open triangles connected with dotted lines, respectively. (b): Experimentally measured MAD in ${ }^{238} \mathrm{U}+{ }^{64} \mathrm{Ni}$ reaction at $E_{\text {c.m. }} \approx$ $302.62 \mathrm{MeV}$. The figure shown in (b) is taken from Ref. [20].

tween TDHF calculations and measurements have been reported for ${ }^{40} \mathrm{Ca}+{ }^{238} \mathrm{U}[57]$ and ${ }^{50,54} \mathrm{Cr}+{ }^{180,186} \mathrm{~W}$ [59].

In Fig. 9 (a), we show the MAD plot in the TDHF calculation, which is constructed from the results shown in Figs. 2 (a) and 4 for ${ }^{64} \mathrm{Ni}+{ }^{238} \mathrm{U}$ reaction at $E_{\text {c.m. }} \approx$ $307.35 \mathrm{MeV}$. In (b), the measured MAD for ${ }^{238} \mathrm{U}+{ }^{64} \mathrm{Ni}$ reaction at $E_{\text {c.m. }} \approx 302.62 \mathrm{MeV}$ is shown. Because the inverse kinematics was employed in the experiment, the angle of $180^{\circ}-\theta_{\text {c.m. }}$. is used in the plot of (a).

As seen from Figs. 2 (a) and 4, both scattering angle and fragment masses change substantially when two nuclei start to overlap in the course of collision. These trends induce correlated behavior in (a) showing an oblique distribution from $A_{\mathrm{L}(\mathrm{H})}=64(238)$ to $A_{\mathrm{L}(\mathrm{H})} \approx$ 100 (200). We note that the TDHF calculation provides no contributions to $\theta_{\text {c.m. }} \lesssim(\gtrsim) 90^{\circ}$ at $A \approx 64$ (238), due to the classical nature of the trajectory.

In our TDHF calculations, collisions at a small- $b$ region in which the mass-drift mode toward the mass symmetry is observed contribute to certain fragment masses. Reactions of the $x$ - and $z$-direction cases produce fragments of $A_{\mathrm{L}} \approx 90-100$ and $A_{\mathrm{H}} \approx 200-210$, while those of the $y$-direction case produce fragments around $A_{\mathrm{L}} \approx 120$ and $A_{\mathrm{H}} \approx 180$. Therefore, we expect that the yields in the MAD of the fragments with symmetric masses are caused by the collisions in the $y$-direction case.

\section{Mass-TKE distribution}

The TKE of outgoing fragments is also a characteristic observable of QF processes. In Fig. 10 (a), we show the mass-TKE distribution in the TDHF calculation, which is constructed from the results shown in Figs. 2 (b) and 4. In (b), the measured mass-TKE distribution for ${ }^{64} \mathrm{Ni}+{ }^{238} \mathrm{U}$ reaction at $E_{\text {c.m. }} \approx 301.05 \mathrm{MeV}$ [19] is shown. In these plots, two prominent peaks at around $A_{\mathrm{L}} \approx 64$ and $A_{\mathrm{H}} \approx 238$ are seen, which correspond to 

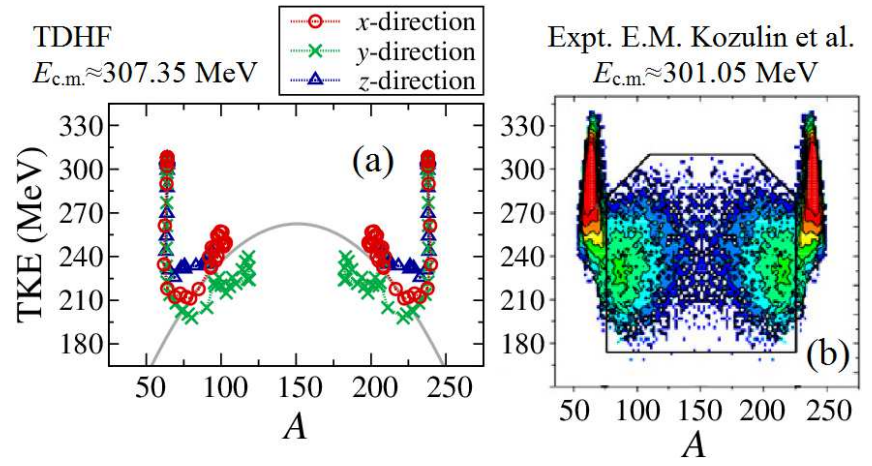

FIG. 10. (Color online) Mass-TKE (total kinetic energy) distribution plots. (a): TDHF results for ${ }^{64} \mathrm{Ni}+{ }^{238} \mathrm{U}$ reaction at $E_{\text {c.m. }} \approx 307.35 \mathrm{MeV}$. Results for $x$-, $y$-, and $z$-direction cases are shown by red circles, green crosses, and blue open triangles connected with dotted lines, respectively. The Viola systematics [94-96] is also shown by a gray solid curve. (b): Experimentally measured mass-TKE distribution for ${ }^{64} \mathrm{Ni}+{ }^{238} \mathrm{U}$ reaction at $E_{\text {c.m. }}=301.05 \mathrm{MeV}$. The figure shown in (b) is taken from Ref. [19].

(quasi)elastic scattering followed by deep-inelastic collisions. Between these two peaks, QF processes characterized by the mass-drift mode toward the mass symmetry accompanying large energy losses are seen.

From the figure, we find a reasonable agreement between the TDHF results and the experimental data. The TDHF results show minima at $A_{\mathrm{L}} \approx 75$ and $A_{\mathrm{H}} \approx 225$ with $\mathrm{TKE} \approx 200$ in the $y$-direction case. In the measurement, we find minima at similar values of masses and TKE in the green areas which correspond to relatively large yields. The mass-TKE distribution in the TDHF calculation extends toward the mass symmetry, up to $A_{\mathrm{L}} \approx 100-120$ and $A_{\mathrm{H}} \approx 180-200$. This trend also reasonably agrees with the experimental data in (b).

Both the mass-TKE distributions obtained from the TDHF calculations and the measurement show a similar trend that the TKE value increases as the system approaches to the mass symmetry. This behavior can be understood by the Viola systematics [94-96], which assumes that the TKE is produced by the Coulomb repulsion at a scission configuration of the composite system. The Viola systematics is shown by a gray solid curve in (a). Similar comparison was reported for side collisions of ${ }^{40} \mathrm{Ca}+{ }^{238} \mathrm{U}$ at $b=0 \mathrm{fm}[58]$.

Looking in detail at results shown in (a), our TDHF results indicate that the $x$-direction case shows smaller dissipation (larger TKE) than the $y$-direction case, as observed in Fig. 2 (b). This may reflect the shell effect of ${ }^{208} \mathrm{~Pb}$, which is important in the $x$-direction case as shown in Fig. 4.

\section{SUMMARY AND CONCLUSION}

In this article, we have reported a detailed investigation of multinucleon transfer (MNT) and quasifission
(QF) processes in ${ }^{64} \mathrm{Ni}+{ }^{238} \mathrm{U}$ reaction within the microscopic framework of the time-dependent Hartree-Fock (TDHF) theory. For this reaction, abundant experimental data are available for both MNT [18] and QF processes $[19,20]$. We compared our TDHF results with the experimental data.

Because ${ }^{238} \mathrm{U}$ is substantially deformed in a prolate shape, we performed TDHF calculations for three initial orientations of ${ }^{238} \mathrm{U}$ : the symmetry axis of ${ }^{238} \mathrm{U}$ is set parallel to the collision axis, set parallel to the impact parameter vector, and set perpendicular to the reaction plane. We have called these three cases as $x$-, $y$-, and $z$-direction cases, respectively.

In all cases, a fast charge equilibration process was observed because of the large $N / Z$ asymmetry (1.29 for ${ }^{64} \mathrm{Ni}$ and 1.59 for $\left.{ }^{238} \mathrm{U}\right)$. In peripheral collisions, MNT processes take place. Several neutrons and protons are transferred toward the direction expected from the initial $N / Z$ ratios. When two nuclei collide deeply at small impact parameters, a dinuclear system connected by a thick neck is formed. When the neck raptures after the charge equilibration in the dinuclear system, the shape evolution dynamics leading to the rapture of the neck characterizes the amount of nucleon transfers. We have regarded the latter process as a QF process, characterized by a large number of transferred nucleons, a large amount of energy loss, and a long contact time.

For the MNT process, we evaluated transfer cross sections using the particle-number projection method and compared them with experimental data [18]. When the number of transferred nucleons is small, the TDHF calculation reasonably describes the process. As the number of transferred protons increases, there is a disagreement between the TDHF calculation and the measurements. The TDHF calculation gives a peak in the MNT cross section at less transferred neutrons than the measurements. Similar success and failure have been observed in lighter systems $[47,54,80,81]$.

In $\mathrm{QF}$ processes, we have found that there appear substantial orientation dependence. In $x$ - $(y$ - $)$ direction case, we have found a larger (smaller) TKE and a shorter (longer) contact time. In the $x$-direction case (close to the tip collision), an elongated dinuclear system is formed. This elongated system tends to split into two fragments in which a heavier fragment is close to ${ }^{208} \mathrm{~Pb}$. This indicates a significant effect of the quantum shells of ${ }^{208} \mathrm{~Pb}$ in the $x$-direction case. The observed larger TKE and shorter contact time for the $x$-direction case is thus expected to be originated from the shell effect of ${ }^{208} \mathrm{~Pb}$ in the QF process. On the other hand, in the $y$-direction case (close to the side collision), a compact dinuclear system is formed. This system tends to split into more masssymmetric fragments, showing less influence of the quantum shells of ${ }^{208} \mathrm{~Pb}$. Instead, we have found an indication of a shell effect of $Z=50$ in the $y$-direction case.

For QF processes, we compared average fragment masses, scattering angle, and total kinetic energy (TKE) of outgoing fragments with experimental data [19, 20]. From the comparison, we have found that the TDHF 
calculations reasonably describe gross behavior seen in the experimental data. We consider that these agreements are noteworthy since no empirical parameters are involved in our calculations.

One of the important applications of the microscopic TDHF theory is to predict optimal conditions to produce objective unstable nuclei, including superheavy elements. One interesting observation in the present study is the occurrence of a capture process in the $y$-direction case at incident energies substantially higher than the Coulomb barrier. To make a reliable prediction, it is important to take account of the effect of particle evaporation in competition with fission employing a statistical model. A study along this direction is in progress [53, 80, 81].

The present study elucidates to what extent the microscopic TDHF theory can describe damped collisions of heavy nuclei at low energies, taking ${ }^{64} \mathrm{Ni}+{ }^{238} \mathrm{U}$ system as an example. The significance of the nuclear structure effects in QF processes is clearly demonstrated for this system. To increase reliability of descriptions for MNT and QF processes, extensions of the theory going beyond the mean-field description are required.

\section{ACKNOWLEDGMENTS}

One of the authors (K.S.) appreciates K. Washiyama and B.J. Roy for giving valuable comments on this article. K.S. acknowledges support of Polish National Science Centre (NCN) Grant, decision no. DEC2013/08/A/ST3/00708. This research used computational resources of the HPCI system provided by Information Initiative Center, Hokkaido University, through the HPCI System Research Projects (Project IDs: hp140010 and hp150081). A part of computation was carried out at the Yukawa Institute Computer Facility. This work was supported by the Japan Society for the Promotion of Science (JSPS) Grants-in-Aid for Scientific Research Grant Numbers 23340113 and 15H03674, and by the JSPS Grant-in-Aid for JSPS Fellows Grant Number 25241.
[1] W. Reisdorf, J. Phys. G: Nucl. Part. Phys. 20, 1297 (1994).

[2] K. Hagino and N. Takigawa, Prog. Theor. Phys. 128, 1061 (2012).

[3] G. Münzenberg, Rep. Prog. Phys. 51, 57 (1988).

[4] S. Hofmann, Rep. Prog. Phys. 61, 639 (1998).

[5] C. H. Dasso, G. Pollarolo, and A. Winther, Phys. Rev. Lett. 73, 1907 (1994).

6] C. H. Dasso, G. Pollarolo, and A. Winther, Phys. Rev. C 52, 2264 (1995).

[7] E.M. Kozulin, E. Vardaci, G.N. Knyazheva, A.A. Bogachev, S.N. Dmitriev, I.M. Itkis, M.G. Itkis, A.G. Knyazev, T.A. Loktev, K.V. Novikov, E.A. Razinkov, O.V. Rudakov, S.V. Smirnov, W. Trzaska, and V.I. Zagrebaev, Phys. Rev. C 86, 044611 (2012).

[8] Y.X. Watanabe, Y.H. Kim, S.C. Jeong, Y. Hirayama, N. Imai, H. Ishiyama, H.S. Jung, H. Miyatake, S. Choi, J.S. Song, E. Clement, G. de France, A. Navin, M. Rejmund, C. Schmitt, G. Pollarolo, L. Corradi, E. Fioretto, D. Montanari, M. Niikura, D. Suzuki, H. Nishibata, and J. Takatsu, Phys. Rev. Lett. 115, 172503 (2015).

[9] V. Zagrebaev and W. Greiner, Phys. Rev. Lett. 101, 122701 (2008).

[10] V. I. Zagrebaev and W. Greiner, Phys. Rev. C 87, 034608 (2013).

[11] V. I. Zagrebaev, B. Fornal, S. Leoni, and W. Greiner, Phys. Rev. C 89, 054608 (2014).

[12] V. I. Zagrebaev, Yu. Ts. Oganessian, M. G. Itkis, and W. Greiner, Phys. Rev. C 73, 031602(R) (2006).

[13] V. Zagrebaev and W. Greiner, Phys. Rev. C 78, 034610 (2008).

[14] Z.-Q. Feng, G.-M. Jin, and J.-Q. Li, Phys. Rev. C 80, 067601 (2009).

[15] L. Zhu, Z.-Q. Feng, and F. S. Zhang, J. Phys. G: Nucl. Part. Phys. 42, 085102 (2015).

[16] M.-H. Mun, G.G. Adamian, N.V. Antonenko, Y. Oh, and
Y. Kim, Phys. Rev. C 89, 034622 (2014).

[17] M.-H. Mun, G.G. Adamian, N.V. Antonenko, Y. Oh, and Y. Kim, Phys. Rev. C 91, 054610 (2015).

[18] L. Corradi, A.M. Stefanini, C.J. Lin, S. Beghini, G. Montagnoli, F. Scarlassara, G. Pollarolo, and A. Winther, Phys. Rev. C 59, 261 (1999).

[19] E.M. Kozulin, G.N. Knyazheva, I.M. Itkis, M.G. Itkis, A.A. Bogachev, L. Krupa, T.A. Loktev, S.V. Smirnov, V.I. Zagrebaev, J. Aysto, W.H. Trzaska, V.A. Rubchenya, E. Vardaci, A.M. Stefanini, M. Cinausero, L. Corradi, E. Fioretto, P. Mason, G.F. Prete, R. Silvestri, S. Beghini, G. Montagnoli, F. Scarlassara, F. Hanappe, S.V. Khlebnikov, J. Kliman, A. Brondi, A. Di Nitto, R. Moro, N. Gelli, and S. Szilner, Phys. Lett. B 686, 227 (2010).

[20] J. Tōke, R. Bock, G.X. Dai, A. Gobbi, S. Gralla, K.D. Hildenbrand, J. Kuzminski, W.F.J. Müller, A. Olmi, H. Stelzer, B.B. Back, and S. Bjørnholm, Nucl. Phys. A440, 327 (1985).

[21] A. Winther, Nucl. Phys. A572, 191 (1994); A594, 203 (1995).

[22] http://personalpages.to.infn.it/ nanni/grazing/

[23] Website of Nuclear Reactions Video (NRV) Project: http://nrv.jinr.ru/nrv/webnrv/grazing/

[24] E. Vigezzi and A. Winther, Ann. Phys. (N.Y.) 192, 432 (1989).

[25] L. Corradi, G. Pollarolo, and S. Szilner, J. Phys. G: Nucl. Part. Phys. 36, 113101 (2009).

[26] R. Yanez and W. Loveland, Phys. Rev. C 91, 044608 (2015).

[27] J.S. Barrett, W. Loveland, R. Yanez, S. Zhu, A.D. Ayangeakaa, M.P. Carpenter, J.P. Greene, R.V.F. Janssens, T. Lauritsen, E.A. McCutchan, A.A. Sonzogni, C.J. Chiara, J.L. Harker, and W.B. Walters, Phys. Rev. C 91, 064615 (2015).

[28] V. Zagrebaev and W. Greiner, J. Phys. G: Nucl. Part. Phys. 31, 825 (2005). 
[29] V. Zagrebaev and W. Greiner, J. Phys. G: Nucl. Part. Phys. 34, 1 (2007).

[30] V. Zagrebaev and W. Greiner, J. Phys. G: Nucl. Part. Phys. 34, 2265 (2007).

[31] N.V. Antonenko, E.A. Cherepanov, A.K. Nasirov, V.P. Permjakov, and V.V. Volkov, Phys. Rev. C 51, 2635 (1995).

[32] G.G Adamian, N.V. Antonenko, and W. Scheid, Nucl. Phys. A618, 176 (1997).

[33] G.G Adamian, N.V. Antonenko, W. Scheid, and V.V. Volkov, Nucl. Phys. A627, 361 (1997).

[34] G.G Adamian, N.V. Antonenko, W. Scheid, and V.V. Volkov, Nucl. Phys. A633, 409 (1998).

[35] G.G. Adamian, N.V. Antonenko, and W. Scheid, Phys. Rev. C 68, 034601 (2003).

[36] G.G. Adamian, N.V. Antonenko, V.V. Sargsyan, and W. Scheid, Phys. Rev. C 81, 024604 (2010).

[37] G.G. Adamian, N.V. Antonenko, V.V. Sargsyan, and W. Scheid, Phys. Rev. C 81, 057602 (2010).

[38] G.G. Adamian, N.V. Antonenko, and D. Lacroix, Phys. Rev. C 82, 064611 (2010).

[39] N. Wang, Z. Li, and X. Wu, Phys. Rev. C 65, 064608 (2002).

[40] N. Wang, Z. Li, X. Wu, J. Tian, Y. Zhang, and M. Liu, Phys. Rev. C 69, 034608 (2004).

[41] J. Tian, X. Wu, K. Zhao, and Y. Zhang, Phys. Rev. C 77, 064603 (2008).

[42] K. Zhao, Z. Li, X. Wu, and Y. Zhang, Phys. Rev. C 88, 044605 (2013).

[43] K. Zhao, Z. Li, N. Wang, Y. Zhang, Q. Li, Y. Wang, and X. Wu, Phys. Rev. C 92, 024613 (2015).

[44] N. Wang, K. Zhao, and Z. Li, Science China Physics, Mechanics \& Astronomy 58, 112001 (2015).

[45] J.W. Negele, Rev. Mod. Phys. 54, 913 (1982).

[46] C. Simenel, Eur. Phys. J. A 48, 152 (2012).

[47] K. Sekizawa and K. Yabana, Phys. Rev. C 88, 014614 (2013); 93, 029902(E) (2016).

[48] L. Corradi, J.H. He, D. Ackermann, A.M. Stefanini, A. Pisent, S. Beghini, G. Montagnoli, F. Scarlassara, G.F. Segato, G. Pollarolo, C.H. Dasso, and A. Winther, Phys. Rev. C 54, 201 (1996).

[49] L. Corradi, A.M. Stefanini, J.H. He, S. Beghini, G. Montagnoli, F. Scarlassara, G.F. Segato, G. Pollarolo, and C.H. Dasso, Phys. Rev. C 56, 938 (1997).

[50] L. Corradi, A.M. Vinodkumar, A.M. Stefanini, E. Fioretto, G. Prete, S. Beghini, G. Montagnoli, F. Scarlassara, G. Pollarolo, F. Cerutti, and A. Winther, Phys. Rev. C 66, 024606 (2002).

[51] S. Szilner, L. Corradi, G. Pollarolo, S. Beghini, B.R. Behera, E. Fioretto, A. Gadea, F. Haas, A. Latina, G. Montagnoli, F. Scarlassara, A.M. Stefanini, M. Trotta, A.M. Vinodkumar, and Y. Wu, Phys. Rev. C 71, 044610 (2005).

[52] C. Simenel, Phys. Rev. Lett. 105, 192701 (2010).

[53] K. Sekizawa and K. Yabana, Phys. Rev. C 90, 064614 (2014).

[54] Sonika, B.J. Roy, A. Parmar, U.K. Pal, H. Kumawat, V. Jha, S.K. Pandit, V.V. Parkar, K. Ramachandran, K. Mahata, A. Pal, S. Santra, A.K. Mohanty, and K. Sekizawa, Phys. Rev. C 92, 024603 (2015).

[55] C. Golabek and C. Simenel, Phys. Rev. Lett. 103, 042701 (2009).

[56] D.J. Kedziora and C. Simenel, Phys. Rev. C 81, 044613 (2010).
[57] A. Wakhle, C. Simenel, D.J. Hinde, M. Dasgupta, M. Evers, D.H. Luong, R. du Rietz, and E. Williams, Phys. Rev. Lett. 113, 182502 (2014).

[58] V.E. Oberacker, A.S. Umar, and C. Simenel, Phys. Rev. C 90, 054605 (2014).

[59] K. Hammerton, Z. Kohley, D.J. Hinde, M. Dasgupta, A. Wakhle, E. Williams, V.E. Oberacker, A.S. Umar, I.P. Carter, K.J. Cook, J. Greene, D.Y. Jeung, D.H. Luong, S.D. McNeil, C.S. Palshetkar, D.C. Rafferty, C. Simenel, and K. Stiefel, Phys. Rev. C 91, 041602(R) (2015).

[60] K. Washiyama, Phys. Rev. C 91, 064607 (2015).

[61] A.S. Umar, V.E. Oberacker, and C. Simenel, Phys. Rev. C 92, 024621 (2015).

[62] K. Sekizawa, Multinucleon Transfer Reactions and Quasifission Processes in Time-Dependent Hartree-Fock Theory, Ph.D. thesis, University of Tsukuba, 2015.

[63] J.W. Eastwood and D.R.K. Brownrigg, J. Compt. Phys. 32, 24 (1979).

[64] E. Chabanat, P. Bonche, P. Haensel, J. Meyer and R. Schaeffer, Nucl. Phys. A635, 231 (1998); A643, 441 (1998).

[65] See Supplemental Material at http://link.aps.org/ supplemental/10.1103/PhysRevC.93.054616 for movies of the ${ }^{64} \mathrm{Ni}+{ }^{238} \mathrm{U}$ reaction with various initial conditions.

[66] K. Nishio, H. Ikezoe, S. Mitsuoka, I. Nishinaka, Y. Nagame, Y. Watanabe, T. Ohtsuki, K. Hirose, and S. Hofmann, Phys. Rev. C 77, 064607 (2008).

[67] K. Nishio, S. Hofmann, F. Heßberger, D. Ackermann, S. Antalic, Y. Aritomo, V. Comas, Ch. Düllmann, A. Gorshkov, R. Graeger, K. Hagino, S. Heinz, J. Heredia, K. Hirose, H. Ikezoe, J. Khuyagbaatar, B. Kindler, I. Kojouharov, B. Lommel, R. Mann, S. Mitsuoka, Y. Nagame, I. Nishinaka, T. Ohtsuki, A. Popeko, S. Saro, M. Schädel, A. Türler, Y. Watanabe, A. Yakushev, and A. Yeremin, Phys. Rev. C 82, 024611 (2010).

[68] K. Nishio, S. Mitsuoka, I. Nishinaka, H. Makii, Y. Wakabayashi, H. Ikezoe, K. Hirose, T. Ohtsuki, Y. Aritomo, and S. Hofmann, Phys. Rev. C 86, 034608 (2012).

[69] E.M. Kozulin, G.N. Knyazheva, S.N. Dmitriev, I.M. Itkis, M.G. Itkis, T.A. Loktev, K.V. Novikov, A.N. Baranov, W.H. Trzaska, E. Vardaci, S. Heinz, O. Beliuskina, and S.V. Khlebnikov, Phys. Rev. C 89, 014614 (2014).

[70] E.M. Kozulin, G.N. Knyazheva, I.M. Itkis, N.I. Kozulina, T.A. Loktev, K.V. Novikov, and I. Harca, Journal of Physics: Conference Series 515, 012010 (2014).

[71] M.G. Itkis, J. Äystö, S. Beghini, A.A. Bogachev, L. Corradi, O. Dorvaux, A. Gadea, G. Giardina, F. Hanappe, I.M. Itkis, M. Jandel, J. Kliman, S.V. Khlebnikov, G.N. Kniajeva, N.A. Kondratiev, E.M. Kozulin, L. Krupa, A. Latina, T. Materna, G. Montagnoli, Yu.Ts. Oganessian, I.V. Pokrovsky, E.V. Prokhorova, N. Rowley, V.A. Rubchenya, A.Ya. Rusanov, R.N. Sagaidak, F. Scarlassara, A.M. Stefanini, L. Stuttge, S. Szilner, M. Trotta, W.H. Trzaska, D.N. Vakhtin, A.M. Vinodkumar, V.M. Voskressenski, and V.I. Zagrebaev, Nucl. Phys. A734, 136 (2004).

[72] E.M. Kozulin, G.N. Knyazheva, I.M. Itkis, M.G. Itkis, A.A. Bogachev, E.V. Chernysheva, L. Krupa, F. Hanappe, O. Dorvaux, L. Stuttgé, W.H. Trzaska, C. Schmitt, and G. Chubarian, Phys. Rev. C 90, 054608 (2014).

[73] Y. Aritomo, Phys. Rev. C 80, 064604 (2009).

[74] Y. Aritomo, K. Hagino, K. Nishio, S. Chiba, Phys. Rev. 
C 85, 044614 (2012).

[75] Y. Aritomo and M. Ohta, Nucl. Phys. A744, 3 (2004).

[76] Y. Aritomo and M. Ohta, Nucl. Phys. A753, 152 (2005).

[77] G. Scamps, C. Simenel, and D. Lacroix, Phys. Rev. C 92, 011602(R) (2015).

[78] M. Morjean, D. Jacquet, J.L. Charvet, A. L'Hoir, M. Laget, M. Parlog, A. Chbihi, M. Chevallier, C. Cohen, D. Dauvergne, R. Dayras, A. Drouart, C. EscanoRodriguez, J.D. Frankland, R. Kirsch, P. Lautesse, L. Nalpas, C. Ray, C. Schmitt, C. Stodel, L. Tassan-Got, E. Testa, and C. Volant, Phys. Rev. Lett. 101, 072701 (2008).

[79] M.O. Frégeau, D. Jacquet, M. Morjean, E. Bonnet, A. Chbihi, J.D. Frankland, M.F. Rivet, L. Tassan-Got, F. Dechery, A. Drouart, L. Nalpas, X. Ledoux, M. Parlog, C. Ciortea, D. Dumitriu, D. Fluerasu, M. Gugiu, F. Gramegna, V.L. Kravchuk, T. Marchi, D. Fabris, A. Corsi, and S. Barlini, Phys. Rev. Lett. 108, 122701 (2012).

[80] K. Sekizawa and K. Yabana, EPJ Web of Conferences 86, 00043 (2015).

[81] K. Sekizawa and K. Yabana, arXiv:1511.08322 [nucl-th].

[82] R. Balian and M. Vénéroni, Phys. Rev. Lett. 47, 1353 (1981).

[83] C. Simenel, Phys. Rev. Lett. 106, 112502 (2011).

[84] S. Ayik, Phys. Lett. B 658, 174 (2008).

[85] K. Washiyama, S. Ayik, and D. Lacroix, Phys. Rev. C 80, 031602(R) (2009).
[86] S. Ayik, K. Washiyama, and D. Lacroix, Phys. Rev. C 79, 054606 (2009).

[87] D. Lacroix, S. Ayik, and B. Yilmaz, Phys. Rev. C 85, 041602(R) (2012).

[88] D. Lacroix, D. Gambacurta and S. Ayik, Phys. Rev. C 87, 061302(R) (2013).

[89] B. Yilmaz, S. Ayik, D. Lacroix, and O. Yilmaz, Phys. Rev. C 90, 024613 (2014).

[90] S. Ayik, B. Yilmaz, and O. Yilmaz, Phys. Rev. C 92, 064615 (2015).

[91] R. Bock, Y. T. Chu, M. Dakowski, A. Gobbi, E. Grosse, A. Olmi, H. Sann, D. Schwalm, U. Lynen, W. Müller, S. Bjørnholm, H. Esbensen, W. Wölfli, and E. Morenzoni, Nucl. Phys. A388, 334 (1982).

[92] R. du Rietz, D. J. Hinde, M. Dasgupta, R. G. Thomas, L. R. Gasques, M. Evers, N. Lobanov, and A. Wakhle, Phys. Rev. Lett. 106, 052701 (2011).

[93] R. du Rietz, E. Williams, D. J. Hinde, M. Dasgupta, M. Evers, C. J. Lin, D. H. Luong, C. Simenel, and A. Wakhle, Phys. Rev. C 88, 054618 (2013).

[94] V. E. Viola, K. Kwiatkowski, and M. Walker, Phys. Rev. C 31, 1550 (1985).

[95] D. J. Hinde, J. R. Leigh, J. J. M. Bokhorst, J. O. Newton, R. L. Walsh, and J. W. Boldeman, Nucl. Phys. A472, 318 (1987).

[96] D. J. Hinde, D. Hilscher, H. Rossner, B. Gebauer, M. Lehmann, and M. Wilpert, Phys. Rev. C 45, 1229 (1992). 